\title{
Bistable switching in nonlinear Bragg gratings.
}

\author{
N. G. R. Broderick \\ Optoelectronics Research Centre, University of Southampton, Southampton, SO17 1BJ, UK. \\ Phone: +44 (0)1703 593144, Fax: +44(0)1703 593142, email: ngb@orc.soton.ac.uk
} (September 3, 1997)

\begin{abstract}
We present a new approach to all-optical switching in nonlinear Bragg gratings. In contrast to previous schemes in which the system remains in the switched state for a short length of time we permanent switch a CW probe from a state of high reflectivity to a low reflectivity state using a short optical pump. Finally we discuss the practicality of the device.

42.65.-k,42.65.Pc,42.70.Qs,42.81.-i
\end{abstract}




\section{INTRODUCTION}

Fibre Bragg gratings (FBGs) are emerging as one of the essential components for the next generation of all-optical networks due to their low insertion losses, narrow bandwidths, and high reflectivities. Furthermore these linear features make Bragg gratings particularly interesting nonlinear devices. Indeed it has been shown that a high power pump pulse can significantly alter the propagation of a weak $\mathrm{CW}$ probe beam as they co-propagate through the grating $[1,2]$. These experiments, which relied on the cross phase modulation effect in fibres [3], showed that it was possible to alter the transmission of a probe by the grating in the presence of the pump. When the pump left the grating the transmission of the probe returned to its initial state. Clearly a system in which the propagation of the pump permanently altered the transmission of a probe would be more desirable having obvious applications in all-optical memory and for packet switching in all-optical networks. Fortunately the transmission of nonlinear Bragg gratings is bistable [4] suggesting that it might be possible to permanently switch a Bragg grating using a pump pulse and indeed we present such a scheme below.

Crucial to this scheme is the nonlinear response of a FBG to an intense CW excitation. Winful et al. showed that the under these conditions the transmission (and reflection) of Bragg grating exhibits bistable behaviour [4]. Fig. 1 shows the transmission of a uniform Bragg grating as a function of the input intensity. Note that for a range of input intensities the transmission of the grating can be in a variety of states. Which particular state it is in depends on the history of the device, which is the essence of this scheme. The experiments discussed previously operated in the low power region of Fig. 1 where only one state exists and hence the initial and final states of these experiments were identical. In contrast I propose to operate at a input intensity near the dashed line in Fig. 1 where the grating is bistable. The initial and final states are given by the two solid dots in Fig. 1. We note that the middle branch of the transmission curve has been shown to be unstable [5] and hence we ignore it in this work. In the next section I present a theoretical model of our switch and 
proceed to show numerically that it can work.

\section{DESCRIPTION OF THE SYSTEM}

A uniform Bragg grating couples forward and backward propagating modes with frequency $\omega$ close to the Bragg frequency $\omega_{0}$, while light at other frequencies can propagate unchanged through the grating. We utilise this by situating the frequency $\omega_{p}$ of the pump pulse far from the Bragg resonance so that the pump does not see the grating while the probe's frequency is assumed to lie within the photonic bandgap of the FBG. The relevant portion of the electric field is then given by

$$
\mathrm{E}(z, t)=\left(f_{+}(z, t) e^{i\left(k_{0} z-\omega_{0} t\right)}+f_{-}(z, t) e^{-i\left(k_{0} z+\omega_{0} t\right)}\right) \hat{\mathbf{x}}+P(z, t) e^{i\left(k_{p} z-\omega_{p} t\right)} \hat{\mathbf{y}}
$$

where $\hat{\mathbf{x}}$, and $\hat{\mathbf{y}}$ are the unit vectors orthogonal to the direction of propagation. $f_{ \pm}$, are the slowly varying envelopes of the forward and backward propagating modes. The pump envelope is given by $P(z, t)$ where $z$ is the propagation direction. Note that we have taken the pump and probe to be orthogonally polarised. Using the slowly varying approximation we can write the coupled mode equations (CME) for $f_{ \pm}$as $[6]$

$$
\begin{array}{r}
i \frac{\partial f_{+}}{\partial z}+\frac{i}{v_{g}} \frac{\partial f_{+}}{\partial t}+\kappa f_{-}+\delta f_{+}+2 \Gamma\left|f_{-}\right|^{2} f_{+}+\Gamma\left|f_{+}\right|^{2} f_{+}+\frac{2}{3} \Gamma|P(z, \dot{t})|^{2} f_{+}=0 \\
-i \frac{\partial f_{-}}{\partial z}+\frac{i}{v_{g}} \frac{\partial f_{-}}{\partial t}+\kappa f_{+}+\delta f_{-}+2 \Gamma\left|f_{+}\right|^{2} f_{-}+\Gamma\left|f_{-}\right|^{2} f_{-}+\frac{2}{3} \Gamma|P(z, t)|^{2} f_{-}=0 .
\end{array}
$$

We have assumed that the pump propagates unchanged through out the fibre, i.e. $P(z, t)=$ $P\left(z-v_{g} t\right)$. For an optical fibre we have $[7,8]$ :

$$
\delta=\frac{\omega-\omega_{0}}{v_{g}}, \quad \Gamma=\frac{4 \pi n_{0}}{\lambda Z} n^{(2)}
$$

$n_{0}$ is the average refractive index of the FBG, $v_{g}$ is the group velocity in the absence of a grating, $\lambda$ is the free space Bragg wavelength and $Z$ the vacuum impedance. The strength of the nonlinearity is given by $n^{(2)}$ which equals $3 \times 10^{-20} \mathrm{~m}^{2} / \mathrm{W}$ in silica [3]. The parameter $\kappa$ measures the strength of the coupling between $f_{+}$and $f_{-}$and is proportional to the 
refractive index modulation depth of the grating. Note that in Eq. (2a) there are three nonlinear terms, the first term is a cross phase modulation term describing the effect of $f_{-}$ on $f_{+}$while the second is the self phase modulation term which is half as strong as expected. The last term describes the effect of the pump on $f_{+}$. The nonlinear terms in Eq. (2b) have a similar explanation.

Throughout the rest of this paper the following boundary conditions apply,

$$
\begin{aligned}
f_{+}(0, t) & =A_{0}, & & f_{-}(0, t)=R(t) A_{0}, \\
f_{+}(L, t) & =T(t) A_{0} & & f_{-}(L, t)=0
\end{aligned}
$$

where $L$ is the length of the grating and $A_{0}$ is the input intensity. The frequency of the input probe is explicit in Eqs. (2) through the terms proportional to $\delta$ and hence is not included in the boundary conditions. The unknown functions $R(t)$ and $T(t)$ are the instantaneous reflection and transmission coefficients for the grating and are determined by solving the CMEs. We note that both $R$ and $T$ can be greater than unity for a period of time if energy is stored in the grating and then released all at once.

The assumption that the pump propagates unchanged through the grating is easily justified when the probe is weak which is not the case here. However the primary effect of the probe on the pump is to introduce a frequency chirp across the pump. This will not significantly alter the pump's intensity profile over the length of the grating and hence we are justified in ignoring the propagation of the pump. This is discussed further in the next section.

In the absence of the pump and for a CW probe Eqs. (2) can be solved exactly for a finite grating [4] giving the bistability curve in Fig. 1. This regime has been extensively studied both theoretically [5] and experimentally [9]. A key result of this work has been to show that the high transmission branch in Fig. 1 is due to a stationary gap soliton being trapped in the grating $[10]$.

Consider now the case when the probe is sufficiently weak that the first two nonlinear terms in Eqs. 2 can be dropped. Eqs. (2) then reduce to the standard equations describing 
pump-probe interactions in a Bragg grating [6]. In the limit that $P(z, t)$ is constant across the grating the CMEs then reduce to the linear equations for a FBG but with an addition frequency shift of $2 / 3 \Gamma|P(t)|^{2}$. Thus in the weak probe, CW pump regime the only effect of the pump is to uniformly shift the entire reflection spectrum of the FBG down in frequency. If this shift is sufficient that the probe's frequency is now at a reflection zero then complete switching has occured.

In contrast to the two regimes described above this paper is concerned with the regime when all of the nonlinear terms in Eqs. (2) are significant. In this regime it is possible to permanently switch the state of the Bragg grating using a strong pump pulse. As analytic solutions to the CMEs in this regime are not available, numerical solutions are necessary. The results of a typical simulation are presented in Fig 2 for the case of a medium intensity CW probe. In this case the grating had a length of $8 \mathrm{~cm}$ and a strength of $\kappa=0.519 \mathrm{~cm}^{-1}$ corresponding to the grating used in previous switching experiments [2]. The input intensity of the probe was take to be $2 \mathrm{GW} / \mathrm{cm}^{2}$ and had a detuning of $0.4 \mathrm{~cm}^{-1}$ which is just inside the bandgap of the grating. The pump has a sech profile with a peak intensity of $20 \mathrm{GW} / \mathrm{cm}^{2}$ and a FWHM of $10 \mathrm{~ns}$. The feasibility of these parameters is discussed later.

In Fig 2 the solid line gives the reflectivity of the grating, while the dashed line shows the pump profile (on a different scale). It is quite clear that prior to the pump the probe's reflection approached unity while after the pump propagated through the grating the reflection is close to zero. The reason for this can be seen Fig. 3 which show the field distributions inside the grating before and after the passage of the probe. The initial field distribution decays approximately exponentially inside the grating leading to the high reflectivity. This field profile is what is expect for light on the low transmission branch of the bistability curve in Fig. 1. After the pump has traversed the grating the field structure is completely different possessing a resonant structure allowing nearly complete transmission. This suggests that a stationary gap soliton has been trapped in the grating which is what is expected theoretically [10] and shows that we are on the upper branch in Fig. 1. Note that the peak of the intensity profile in Fig. 3b is slightly off-centre resulting in a non-zero reflectivity. 
To understand these results it is helpful to compare them to the CW switching results for a weak probe. As described previously the only effect of the pump in such a system is to change the effective detuning of the probe so that instead of being within the photonic bandgap it lies in a transmission resonance instead. The shape of the field structure for the first linear transmission resonance is very similar to that of the final state shown in Fig. 3 b. Returning to the complete system, the.pump again shifts the detuning of the probe to lie outside the bandgap in a transmission resonance. In Fig. 2 this simple switching accounts for the first dip in the reflection near $t=-5$ and the subsequence increase in reflection suggests that the peak pump power shifts the probe past the first transmission zero. However now the intensity of the probe is sufficient that as the additional detuning caused by the pump is slowly decreased the field profile can smoothly transform itself into a stationary gap soliton. In Fig. 2 this process occurs from $t=0$ to approximately $t=10$. A similar transformation was observed by Kahn et al. who observed that the linear resonances outside the grating smoothly transformed themselves into gap solitons as the incident power increased [11]. Also work by Broderick et al. [12] showed that a linear resonance in a nonuniform Bragg grating could be transformed into a moving gap soliton.

Numerical simulations with different parameters show that permanent switching can be achieved for a reasonable range of parameters. Fixing the power of the probe and altering the detuning reveals a variety of different behaviour from permanent stable switching to unstable transient switching. Between the two regimes the reflectivity remains small after the passage of the pump but oscillates about a centre value with a fixed period. The reason for this behaviour is not fully understood but would appear to be related to the stability of the system, which is discussed further below. However there does appear to be a sufficiently wide window of operation to allow permanent all-optical switching to be easily seen. 


\section{DISCUSSION}

In the previous section we showed numerically that it is possible to permanently switch a Bragg grating from one state to another via the cross phase modulation of a strong pump. Throughout the proceeding sections we have assumed that this interaction takes place in a fibre geometry as previously most work on nonlinear Bragg gratings have concentrated on fibre Bragg gratings. However as the numbers used in simulations show that the power requirements for the probe are currently unavailable while the pump pulse is only just feasible. These power requirements could be reduced by the use of Chalcogenide fibres whose nonlinearity is about 100 times that of silica [13]. In addition the required probe power can be made arbitrarily small by increasing the length of the grating (currently one metre gratings are feasible). However the price for this would be to increase the peak pump power needed and some trade off between decreased probe power and increased pump power would be necessary. By using long gratings in Chalcogenide fibres it should be possible to reduce the probe power needed to $\approx 100 \mathrm{~mW}$. Such power levels are currently available using commercially available laser diodes suggesting that a realisation of this scheme in the near future is possible.

It should be noted that although we have followed convention in referring to Bragg gratings as bistable devices it has not been shown that the upper branch of the bistability curve is in fact stable. However numerical simulations suggest that in some regimes this branch is stable, although in other regimes modulational instability leads to the formation of a periodic train of gap solitons [14]. This instability explains why in some cases the Bragg grating returns to its initial state after the passage of the pump beam.

In the simulations discussed here the pump's width was much longer than the length of the grating and thus the only effect of the pump is to slowly change the effective detuning of the probe beam. Furthermore the long time scales over which the switching takes place suggest that alternative stronger nonlinearities could be used which typically are much slower than the Kerr nonlinearity. This has the additional advantage of reducing the effects 
of modulational instability on our system [14] thus allowing a larger window of operation. For example in a semiconductor geometry the nonlinearity is provided by the presence of free carriers i.e. electrons and has a typical responds time of about 1 ns. Switching powers as low as a few milliwatts have been reported in such geometries [15] making the proposed device practical. An additional attraction of a semiconductor geometry with a free carrier nonlinearity is that in such a system an optical pump could be replaced with an electronic injection of carriers into the device thus providing a high contrast electro-optic switch. Alternatively the grating could be side illuminated by an intense UV beam to create the appropriate number of free carriers. This would eliminate any concern about the effects of propagation on the pump profile.

\section{CONCLUSION}

We have proposed a novel high power nonlinear switch based on a nonlinear Bragg grating and a strong pump pulse. In contrast to previous schemes based on Bragg gratings the pump pulse permanent switches the grating from a high reflectivity to a low reflectivity state making it ideal for applications such as all-optical memory storage and high power all-optical switches. Although currently such a switch is impractical in a silica fibre geometry the powers required are feasible in materials with a slower but stronger nonlinearity. Furthermore in a semiconductor geometry it should be possible to replace the optical pump with an electrical signal thus creating a novel electro-optical switch. 


\section{REFERENCES}

[1] S. LaRochelle, Y. Hibino, V. Mizrahi, and G. I. Stegeman, Elect. Lett. 26, 1459 (1990).

[2] N. G. R. Broderick et al., OSA Topical Meeting on Bragg Gratings, Photosensitivity, and Poling in Glass Fibres and Waveguides Williamsburg, Virginia, October 1997.

[3] G. P. Agrawal, Nonlinear Fibre Optics (Academic Press, San Diego, 1989).

[4] H. G. Winful, J. H. Marburger, and E. Garmire, Applied Physics Letters 35, 379 (1979).

[5] C̀. M. de Sterke and J. E. Sipe, in Progress in Optics, edited by E. Wolf (North Holland, Amsterdam, 1994), Vol. XXXIII, Chap. III Gap Solitons, pp. 203-260.

[6] C. M. de Sterke, Optics Letters 17, 914 (1992).

[7] J. E. Sipe, L. Poladian, and C. M. de Sterke, J. Opt. Soc. Am A 11, 1307 (1994).

[8] C. M. de Sterke and J. E. Sipe, Phys. Rev. A 42, 550 (1990).

[9] N. D. Sankey, D. F. Prelewitz, and T. F. Brown, Appl. Phys. Lett. 60, 1427 (1992).

[10] W. Chen and D. L. Mills, Phys. Rev. Lett. 58, 160 (1987).

[11] L. M. Kahn, K. Huang, and D. L. Mills, Phys. Rev. B 39, 12449 (1989).

[12] N. Broderick, C. M. de Sterke, and J. E. Sipe, Op. Comm. 113, 118 (1994).

[13] M. Asobe, Opt. Fib. Tech. 3, 142 (1997).

[14] H. G. Winful and G. D. Cooperman, Appl. Phys. Lett. 40, 298 (1982).

[15] B. Acklin, M. Cada, J. He, and M. A. Dupertius, Appl. Phys. Lett. 63, 2177 (1993). 


\section{FIGURES}

FIG. 1. Nonlinear transmission for a uniform Bragg grating. Note that for a range of input intensities the transmission is multi-valued. The dashed line indicates the region in which the device operates. The two solid dots show the initial and final states of the Bragg grating.

FIG. 2. Reflectivity of a Bragg grating showing the permanent switching caused by the pump pulse. The dashed line shows the pump profile on a different scale. The parameters for this simulation are giverf in the text.

FİG. 3. Field distributions inside the grating before (a) and after (b) the presence of the pump. The solid line represents the total intensity $\left|f_{+}\right|^{2}+\left|f_{-}\right|^{2}$. The dotted line gives $\left|f_{+}\right|^{2}$ while the dashed line gives $\left|f_{-}\right|^{2}$. Note that in (a) the field decays approximately exponentially leading to the high reflectivity while in (b) a resonance structure has appeared leading to almost complete transmission. 


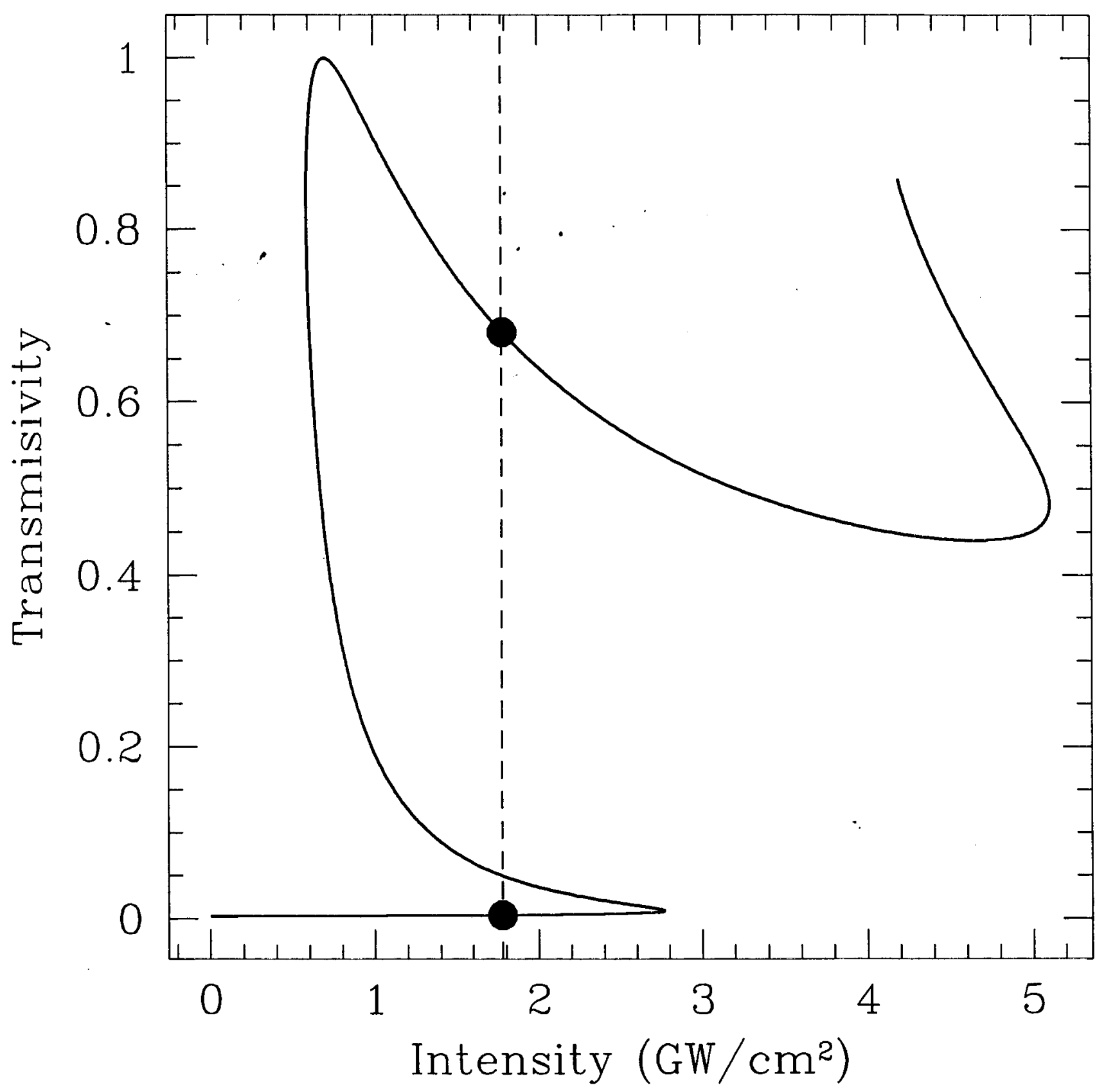




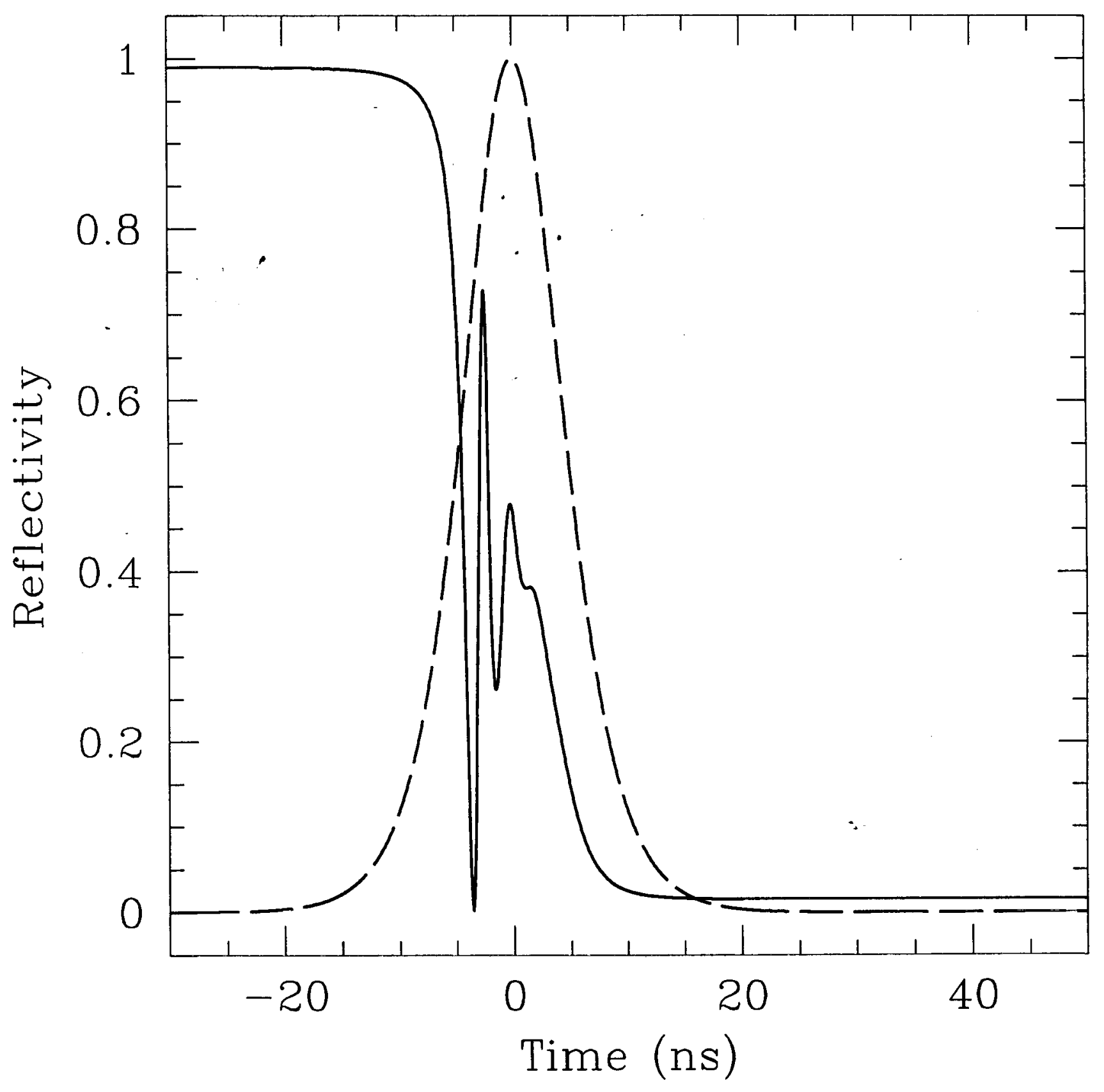



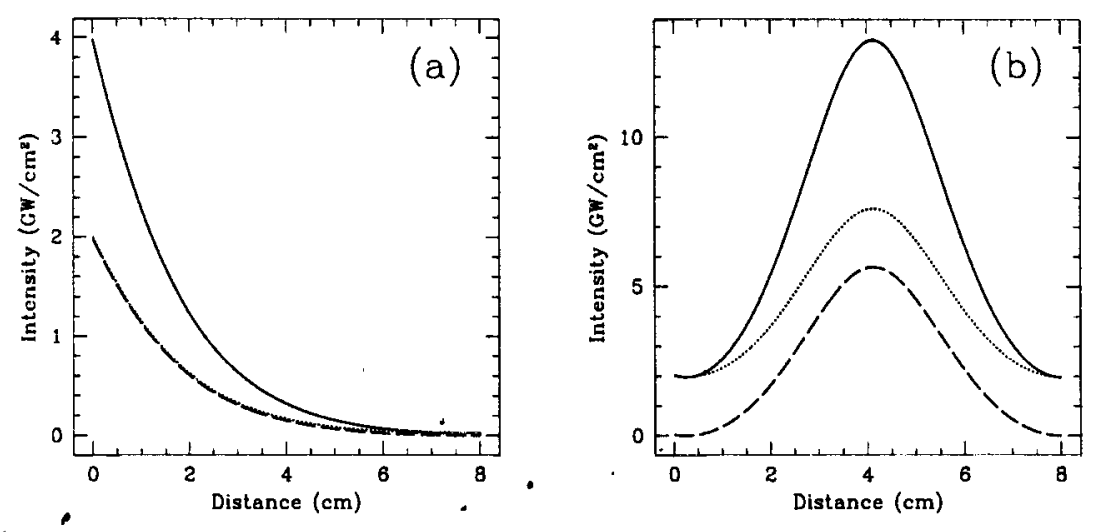